Abanico Agroforestal. Enero-Diciembre 2021; 3:1-15. http://dx.doi.org/10.37114/abaagrof/2021.4 Artículo Original. Recibido: 24/03/2020. Aceptado: 17/06/2021. Publicado: 24/06/2021. Clave:2021-4.

\title{
Caracterización de unidades de producción lechera en el Valle de Tulancingo, Hidalgo, México
}

\section{Characterization of dairy production units in the Tulancingo Valley, Hidalgo, Mexico}

\section{Ruiz-Ortega Maricela ${ }^{11 \mathrm{D}}$, Andrade-Castillo Javier ${ }^{11 \mathrm{D}}$, Cortés-Hernández José ${ }^{1 \mathrm{ID}}$, Ortega-Soto Crystofer ${ }^{11 \mathrm{D}}$, Vigueras-Pérez Misael ${ }^{11 \mathrm{D}}$, Ponce-Covarrubias José ${ }^{2^{*} \mid \mathrm{D}}$}

1 Instituto de Ciencias Agropecuarias, Universidad Autónoma del Estado de Hidalgo, Tulancingo de

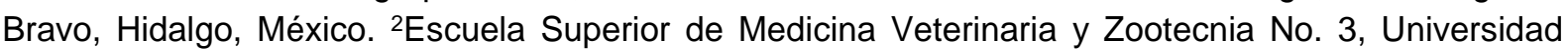
Autónoma de Guerrero (UAGro), Tecpan de Galeana, Guerrero, México. *Autor de correspondencia: José Luis Ponce Covarrubias. Carr. Acapulco-Zihuatanejo km. 106+9000 Col. Las Tunas, C.P. 40900, Tecpan de Galeana, Guerrero, México.maricela_ruiz@uaeh.edu.mx, an347926@uaeh.edu.mx, jechmvz2710@gmail.com,cryss.b2k@gmail.com,mvp.misael@gmail.com,jlponce@uagro.mx

\section{RESUMEN}

El objetivo del presente estudio fue conocer las características socioeconómicas de los productores de ganado bovino lechero, así como, caracterizar las unidades de producción (UP) en el Valle de Tulancingo, Hidalgo, México. Durante los meses de junio y julio del 2020 fue aplicada una encuesta aleatoria a 30 productores de ganado bovino. Con el programa estadístico SAS, fueron analizadas variables cuantitativas, porcentajes y tablas de frecuencia absoluta. Los resultados del estudio muestran que el $39.3 \%$ de los productores tienen una edad entre los 20 a 30 años, el $82.9 \%$ pertenece al sexo masculino con estudios de primaria (28.6\%) y el $35.7 \%$ con estudios superiores. Los productores alimentan a su ganado con forrajes y pre-mezclas $(50 \%)$, y coinciden que las principales enfermedades que afectan a su ganado son la mastitis (82.1\%) y Brucelosis (10.7\%). Los datos respecto a la asesoría técnica indican que el $60.7 \%$ de los productores no han recibido ningún tipo de asesoría. En el caso de programas sociales el $92.9 \%$ de la población encuestada especifica que no ha participado en este tipo de convocatorias. A su vez el $71.4 \%$ desconoce los requisitos para participar en programas ganaderos. Se concluye que los productores de bovinos de leche del Valle de Tulancingo, aunque sean productores a baja escala requieren de asesoría técnica e información sobre diferentes fuentes de apoyo.

Palabras clave: Sistemas, Bovinos, Holstein, Tulancingo.

\begin{abstract}
The aim of this study was to know the socioeconomic characteristics of dairy cattle producers, as well as to characterize the production units (PU) in the Tulancingo Valley, Hidalgo, Mexico. During the months of June and July 2020, a random survey was applied to 30 cattle producers. With the SAS statistical program, quantitative variables, percentages and absolute frequency tables were analyzed. The results of the study showed that $39.3 \%$ of the producers are between 20 and 30 years old, $82.9 \%$ are male with primary education $(28.6 \%)$ and $35.7 \%$ with higher education. The producers feed their cattle with forages and premixes $(50 \%)$, and they agree that the main diseases that appreciate their cattle are mastitis (82.1\%) and Brucellosis (10.7\%). The data regarding technical advice where $60.7 \%$ of the producers have not received any kind of advice. In the case of social programs, $92.9 \%$ of the surveyed population specifies that they have not participated in this type of call. In turn, $71.4 \%$ do not know the requirements to participate in livestock programs. It is concluded that dairy cattle producers in the Tulancingo Valley, even if they are small-scale producers, require technical advice and information on different sources of support.
\end{abstract}

Keywords: Systems, Bovines, Holstein, Tulancingo. 


\section{INTRODUCCIÓN}

La ganadería bovina es una de las principales actividades del sector agropecuario en México, debido a la contribución de productos cárnicos y participación en la balanza comercial del País. Actualmente, la micro empresa bovina presenta desafíos enormes, entre los que destacan las fuentes de financiamiento, su organización, y canales de comercialización escasos (Carrera y Bustamante, 2013). En el estado de Hidalgo, ubicado en la región centro-oriental de México, la producción de leche es una de las actividades pecuarias importantes. En el periodo 1996-2005 se produjeron, en promedio, más de un millón de litros por día, lo que representó el 4.2\% de la producción nacional (SAGARPA, 2006; Cervantes-Escoto et al., 2013).

Ante esta situación es importante realizar la caracterización de unidades de producción, lo cual es determinante para el desarrollo de políticas, ya que permite conocer la conformación de los sistemas de producción, sus componentes tecnológicos, el potencial y limitantes respecto a otros sistemas (Vilaboa y Díaz, 2009). Coronel y Ortuño (2005), señalan que la clasificación adecuada de los sistemas productivos ayuda a conocer la dinámica de desarrollo de una región o al diseño y gestión de proyectos de desarrollo (Méndez-Cortés, 2019).

Los retos que están enfrentado los microempresarios de sistemas de producción de bovinos de leche oscilan entre periodos intensos de sequía, falta de liquidez y recientemente, movilidad reducida (Lara-Rodríguez y Vázquez-Luna, 2020). Cada uno de los sistemas de producción de bovinos de leche se distingue por su heterogeneidad productiva, tanto en las formas de producción como en los diversos tamaños de las unidades productivas; se produce leche tanto en el altiplano como en las zonas tropicales y áridas, bajo condiciones muy distintas (Espinosa et al., 2007), por lo que la resulta importante dar continuidad con los estudios de caracterización en las diferentes zonas de producción lechera.

Las investigaciones sobre caracterización de UP bovina son insuficientes al considerar el tamaño de la unidad o la cantidad de recursos económicos que se invierten en la misma, deben considerar la forma de manejo del sistema de producción, la racionalidad que se le imprime al desarrollo de la actividad, la concepción en el uso de los recursos y las diferencias impuestas por las pautas culturales. Aun cuando los productores pertenezcan a un mismo estado o región, estos no desarrollan su actividad en igualdad de condiciones sociales, económicas y tecnológicas (Granados-Rivera, 2018; Vilaboa y Díaz, 2009). En la caracterización de un "establecimiento ganadero promedio", es importante considerar no sólo las condiciones productivas, sino también las sociales, culturales, económicas y/o ambientales de cada región (Solano et al., 2001).

En el estado de Hidalgo, la producción de leche juega un papel fundamental como medio de ingresos para los productores principalmente de zonas rurales, en la entidad se han desarrollado tres cuencas lecheras importantes: Tizayuca, Valle de Tulancingo y Mezquita (Cervantes-Escoto et al., 2013). Sin embargo, dada la 
variabilidad de las condiciones climatológicas del Estado, las explotaciones ganaderas adquieren características propias por región, influyendo adicionalmente la idiosincrasia, tradición y costumbres de la población (Arias et al., 2008), esto hace necesario realizar mayores esfuerzos por entender el proceso productivo y comercial de la ganadería hidalguense.

Por lo anterior, el objetivo del presente estudio fue conocer las características socioeconómicas de los productores de ganado bovino lechero, así como, caracterizar las unidades de producción (UP) en el Valle de Tulancingo, Hidalgo, México.

\section{MATERIAL Y MÉTODOS}

\section{General}

El presente estudio se realizó en el Valle de Tulancingo, Hidalgo, México. El lugar se localiza geográficamente entre los paralelos $20^{\circ} 03^{\prime}$ y $20^{\circ} 13^{\prime}$ de latitud norte, los meridianos $98^{\circ} 14^{\prime}$ y $98^{\circ} 31^{\prime}$ de longitud oeste y presenta una altitud entre 2200 y 2 $700 \mathrm{msnm}$. La región presenta temperaturas promedio anual de $13.5^{\circ} \mathrm{C}$ y precipitaciones de $1669 \mathrm{~mm}$.

El trabajo se realizó con la participación de la "Asociación Ganadera Local Especializada en Bovinos Productores de Leche del Valle de Tulancingo". Debido a que durante el periodo de estudio México y el mundo se encontraban en pandemia por el efecto del COVID-19, se estableció el siguiente protocolo de trabajo: a) Reconocimiento, identificación y aceptación de los productores, por vía telefónica, cada productor fue llamado para solicitar su autorización y participación en el estudio; b) elaboración previa del cuestionario; d) aplicación de cuestionarios, utilizando la tecnología formularios "GOOGLE". Se realizó una llamada, en el día y hora que cada productor especificó, las respuestas se capturaron directamente en los formularios de forma individual por los encuestadores y e) el análisis descriptivo de los datos.

Todas las preguntas fueron registradas de acuerdo al consentimiento de los productores. Los encuestadores, de acuerdo a los lineamientos para los servicios de ética institucional que responden al COVID-19, permanecieron en casa, debido a que la encuesta se realizó durante el periodo de pandemia (Berlinger et al., 2020).

\section{Diseño del cuestionario y aplicación de la encuesta}

Durante los meses de junio y julio del año 2020 fueron entrevistados aleatoriamente 30 productores de ganado bovino de leche dispersos por el Valle de Tulancingo. Para el estudio se aplicó la fórmula propuesta por Otzen y Manterola (2017), donde la muestra se delimitó utilizando un muestreo aleatorio simple y se consideró a cada Unidad de Producción (UP) como una unidad experimental representada por cada productor ganadero. El cuestionario se construyó con 21 ítems y dividido en cinco apartados: datos del propietario, datos de la unidad de producción, descripción de la producción actual y características de la UP. Las variables de estudio se clasificaron 
en: socioeconómicas, tecnológicas y comerciales, el tipo de variables utilizadas en esta investigación fueron las categóricas y numéricas (Agresti, 2013).

Las variables numéricas, analizadas fueron: edad del productor (años), escolaridad (años), total de bovinos, total de vacas en producción, precio de la leche (\$/litro), producción diaria de leche, edad del becerro a la venta (meses), peso del becerro a la venta $(\mathrm{kg})$, precio de venta del becerro $(\$ \mathrm{~kg} / 1)$, precio de venta de la becerra $(\$$ $\mathrm{kg} / 1)$, precio de venta del torete $(\$ \mathrm{~kg} / 1)$, precio de venta de la vaquilla $(\$ \mathrm{~kg} / 1)$, precio de venta de la vaca $(\$ \mathrm{~kg} / 1)$, precio de venta del toro $(\$ \mathrm{~kg} / 1)$ y porcentaje del ingreso de la leche (\%).

Las variables categóricas incluyeron: tenencia de la tierra, si pertenece a la asociación, programa de gobierno recibido, municipios, unidad de producción ganadera. instalaciones, otros cultivos de siembra, raza, ordeña, principales enfermedades de los bovinos y problemas de venta.

\section{Análisis estadístico}

Para el análisis de la información se empleó estadística descriptiva; mediante tablas de frecuencia se determinó la participación porcentual de cada variable en la población. La información recolectada fue registrada en una hoja electrónica de Microsoft Excel (2016) para su organización y control. La base de datos correspondiente a la identificación de los sistemas de producción de leche fue procesada estadísticamente mediante un análisis multivariado (conglomerado) para su agrupación y clasificación. El análisis estadístico de la información se realizó por medio del software SAS versión 9.0 (Copyright@ 2002 by SAS Institute Inc., Cary, NC, USA).

\section{RESULTADOS}

\section{Caracterización socioeconómica de productores lecheros}

Los análisis de estadísticos descriptivos mostraron que las variables seleccionadas eran independientes y apropiadas para explicar los conjuntos que se agruparon. La primera categoría corresponde a la edad, sexo, grado de escolaridad y años dedicados a la UP de los productores (Tabla 1).

Los productores del Valle de Tulancingo cuentan con una edad entre los 20 y 40 años, de los cuales el $82.1 \%$ pertenece al sexo masculino y el resto femenino. El $28.6 \%$ cuenta con estudios de primaria y el $35.7 \%$ con estudios superiores. El $42.9 \%$ de los productores llevan dedicándose a esta actividad entre 6 y 15 años.

El porcentaje de tipo de propiedad con que cuentan los productores fue de $57.1 \%$ de tipo privada, con una a tres hectáreas $(57.1 \%)$, de estas el $42.9 \%$ de las tierras se encuentran sembradas de pastos y el $67.9 \%$ cuentan con menos de tres personas encargadas del establo (Tabla 2). 
Tabla 1. Datos generales de productores de ganado bovino lechero del Valle de Tulancingo

\begin{tabular}{|c|c|c|}
\hline Variable & Categoría & Porcentaje \\
\hline \multirow[t]{4}{*}{ Edad } & Entre los 20 y 30 años & 39.3 \\
\hline & Entre los 31 y 40 años & 39.3 \\
\hline & Entre los 41 y 50 años & 10.7 \\
\hline & Mayor de 51 años & 10.7 \\
\hline \multirow[t]{2}{*}{ Sexo } & Femenino & 17.9 \\
\hline & Masculino & 82.1 \\
\hline \multirow[t]{5}{*}{ Grado de escolaridad } & Sin estudios & 10.7 \\
\hline & Primaria & 28.6 \\
\hline & Secundaria & 25 \\
\hline & Medio superior & 0 \\
\hline & Superior & 35.7 \\
\hline \multirow[t]{4}{*}{ Años dedicados a la UP } & Menos de 5 años & 21.4 \\
\hline & Entre 6 y 15 años & 42.9 \\
\hline & Entre 16 y 25 años & 10.7 \\
\hline & Más de 25 años & 25 \\
\hline
\end{tabular}

Tabla 2. Características generales de las unidades de producción de bovinos lecheros en el Valle de Tulancingo

\begin{tabular}{llr}
\hline \multicolumn{1}{c}{ Variable } & \multicolumn{1}{c}{ Categoría } & Porcentaje \\
\hline Tipo de propiedad de la UP & Privada & 57.1 \\
& Ejidal & 42.9 \\
Personal encargado de la UP & Menos de tres personas & 67.9 \\
& Entre 4 y 6 personas & 32.1 \\
Hectáreas de la UP & Menos de una hectárea & 14.3 \\
& Entre una y tres hectáreas & 57.1 \\
Superficie de pastos & Más de cuatro hectáreas & 28.6 \\
& Sin superficie de pastos & 17.9 \\
& Menos de una hectárea & 25 \\
& Entre una y tres hectáreas & 42.9 \\
& Más de cuatro hectáreas & 14.3 \\
\hline
\end{tabular}




\section{Caracterización de UP bovinas lecheras}

Se observa que las UP cuentan con 11 y 20 animales (32.1\%), de los cuales se cuenta con menos de 10 machos, ganado con cruzas de Holstein (46.4\%); y el $39.3 \%$ de las vacas se encuentran en producción sometidas a ordeño una vez al día en la mayoría de UP (85.7\%) (Tabla 3).

Referente al tipo de alimentación ( $50 \%$ forrajes y pre-mezclas) y enfermedades (82.1\% presentan mastitis y $10.7 \%$ de Brucelosis) que presentan las vacas, el $75 \%$ de los productores prefiere que la atención medica de su ganado este a cargo de un médico veterinario (Tabla 3 ).

Tabla 3. Características del hato en las UP de bovinos lecheros del Valle de Tulancingo

\begin{tabular}{|c|c|c|}
\hline Variable & Categoría & Porcentaje \\
\hline \multirow[t]{4}{*}{ Número de animales } & Menos de 10 & 25 \\
\hline & Entre 11 y 20 & 32.1 \\
\hline & Entre 21 y 40 & 25 \\
\hline & Más de 41 & 17.9 \\
\hline \multirow[t]{4}{*}{ Vacas en producción } & Menos de 10 & 46.4 \\
\hline & Entre 11 y 20 & 39.3 \\
\hline & Entre 21 y 40 & 3.6 \\
\hline & Más de 41 & 10.7 \\
\hline \multirow[t]{2}{*}{ Machos } & Menos de 10 & 85.7 \\
\hline & Entre 11 y 20 & 14.3 \\
\hline \multirow[t]{3}{*}{ Numero de ordeños al día } & Una vez & 85.7 \\
\hline & Dos veces & 10.7 \\
\hline & Tres Veces & 3.6 \\
\hline \multirow[t]{3}{*}{ Razas } & Holstein & 42.9 \\
\hline & Cruza con Holstein & 46.4 \\
\hline & Jersey & 10.7 \\
\hline \multirow[t]{3}{*}{ Principales enfermedades } & Mastitis & 82.1 \\
\hline & Brucelosis & 10.7 \\
\hline & Otras & 7.2 \\
\hline \multirow[t]{4}{*}{ Tipo de alimentación } & Mezcla de forrajes & 50 \\
\hline & Concentrado & 17.9 \\
\hline & Silo & 14.3 \\
\hline & Otros & 17.9 \\
\hline
\end{tabular}


Respecto a la ordeña el $50 \%$ de las UP utiliza una máquina de ordeño y el resto ordeña manualmente, siendo responsable de esta actividad entre dos y cinco trabajadores (57.1\%) (Tabla 4).

También se muestran los datos respecto a la asesoría técnica en donde el $60.7 \%$ de los productores no han recibido ningún tipo de asesoría. En el caso de programas sociales el $92.9 \%$ de la población encuestada específica que no ha participado en este tipo de convocatorias. A su vez el $71.4 \%$ desconoce los requisitos para participar en programas ganaderos. La asistencia técnica que recibieron las UP estudiadas permitió conocer las principales enfermedades que afectan a los bovinos (Tabla 4).

Las encuestas aplicadas en el presente estudio arrojan que los productores solicitan temas de capacitación relacionados en primer lugar con la reproducción animal (39.3\%) y en segundo lugar con la medicina animal (35.7\%) (Tabla 4).

Tabla 4. Manejo y perspectivas de requerimientos en productores de bovinos lecheros para el Valle de Tulancingo

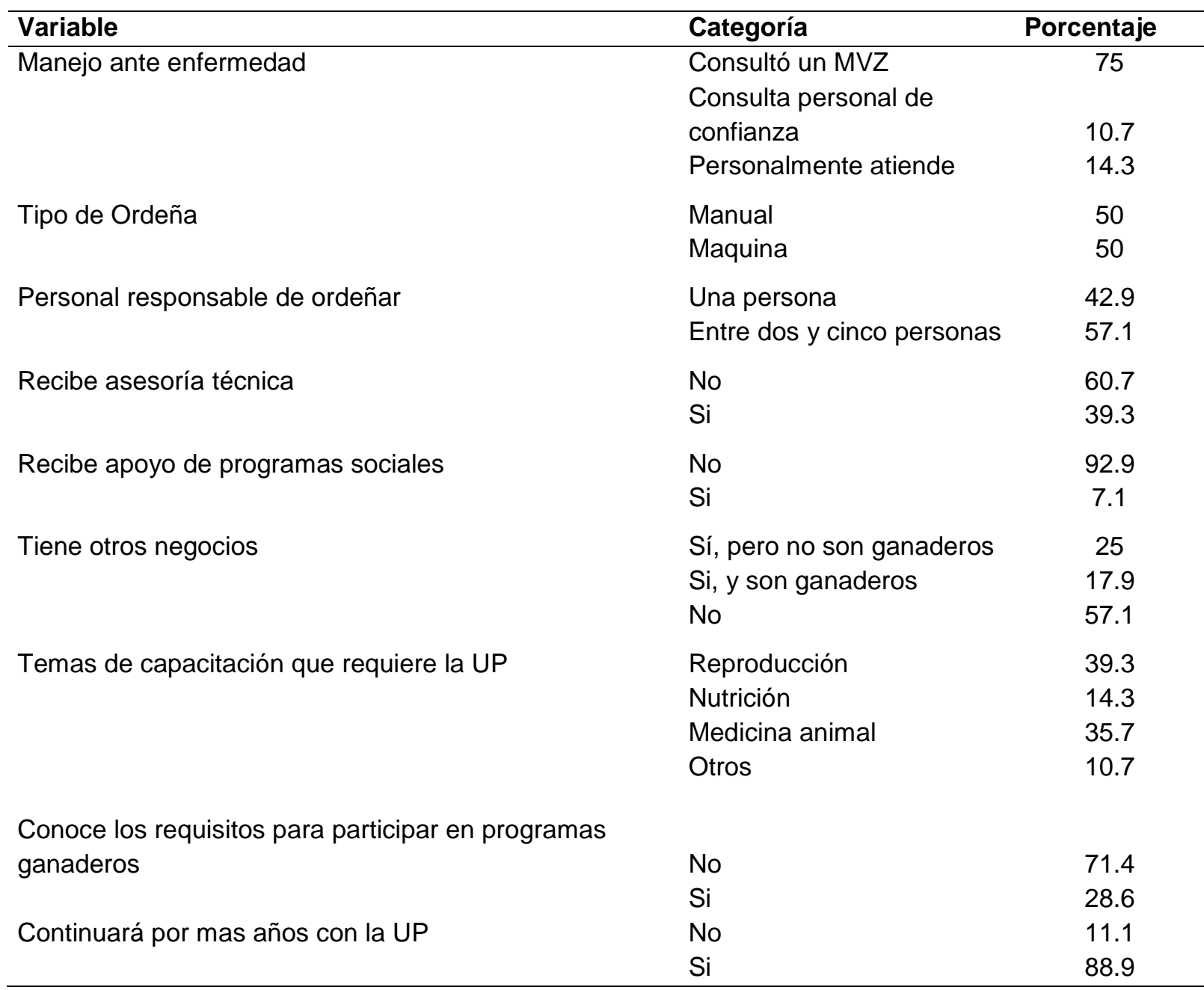


El $50 \%$ de los productores no cuentan con otros negocios aparte de las UP, lo que implica que su actividad principal es la venta de leche. Por otra parte, el $25 \%$ confirmó que cuenta con otros negocios pero que no pertenecen al sector ganadero, mientras que el $17.1 \%$ cuenta con negocios relacionados con la ganadería.

Con respecto a la comercialización de los becerros producidos en la UP, los productores afirman que la venta no es viable debido al bajo precio y mala calidad de la carne, por lo que los mantienen en el hato hasta que otro productor los compra, otro estudio reporta que esta depende fundamentalmente de la participación de intermediarios (98\%).

\section{DISCUSIÓN}

El presente estudio muestra que los ganaderos son adultos y cuentan con educación básica (primaria y secundaria). Solo el $35.7 \%$ cuenta con estudios superiores. También, indican que suplementan a su ganado y tienen identificadas las principales enfermedades presentes en su UP, lo anterior expresa que debe ser un médico veterinario quien se encargue de la sanidad del ganado.

\section{Caracterización socioeconómica de productores lecheros}

Los resultados obtenidos, coinciden con el estudio de caracterización de UP en el estado de Veracruz de Méndez-Cortés (2019) quienes reportan que el 97\% fueron hombres y el $3 \%$ mujeres, con edad promedio ambos de 58 años, superior a lo encontrado en Sinaloa, y Veracruz (Juárez-Barrientos et al., 2015). La edad de los productores de la UP fue similar a la registrada en los estados de Morelos (ChalateMolina et al., 2010), Sinaloa (Cuevas et al., 2012) y Veracruz (Juárez-Barrientos et al., 2015; Oros et al., 2011). La edad del productor es importante debido a que productores de la tercera edad no permiten una adopción de prácticas tecnológicas nuevas en la UP (Fuentes et al., 2012). Por su parte, Granados-Rivera (2018) en su estudio para el estado de Tabasco, menciona que la edad promedio de los productores fue de 54.5 años. En los productores con edad avanzada se reduce la probabilidad de que estos adopten nuevas tecnologías en las UP (Salas-González et al., 2013).

En esta investigación el 35.7\% de los productores del Valle de Tulancingo cuentan con estudios superiores, lo cual fue similar a lo reportado en Veracruz en donde para la variable escolaridad el $22 \%$ contaban con educación superior (Méndez-Cortés, 2019). Por su parte, en el estado de tabasco se reportó que el $52 \%$ de los productores encuestados tuvieron un nivel escolar de primaria y $21 \%$ cuenta con estudios de licenciatura (Granados-Rivera, 2018), lo cual coincide con los productores del Valle de Tulancingo en donde el 56.3\% cuentan con primaria y/o secundaria. El nivel de escolaridad influye directamente en la disponibilidad de los productores para adoptar nuevas tecnologías para la producción (Fuentes et al., 2012; Galindo-González, 2001). 
En el presente estudio se encontró un $42.9 \%$ de productores que se han dedicado a la cría de ganado bovino lechero de entre 6 y quince años. Al respecto, MéndezCortés (2019) reportan una antigüedad de los ganaderos en promedio de 23 años, lo que manifiesta que en su mayoría son productores con años de experiencia en la ganadería.

El $57.1 \%$ de los productores del Valle de Tulancingo encuestados indican que la tenencia de sus tierras es de tipo privada, lo cual no coinciden con el estudio de Granados-Rivera (2018) quien reporta una tenencia de la tierra de tipo ejidal en $74 \%$ y de tipo particular en $26 \%$. En otras investigaciones la tenencia de la tierra es de tipo ejidal mayoritariamente, mientras que las UP localizadas en la región de Las Choapas, Veracruz y Centro de Chiapas, México la tenencia de la tierra es principalmente de tipo particular (Díaz-Rivera et al., 2011; Orantes-Zebadúa et al., 2014). EL 67.9\% de las encuestas de nuestro estudio indican que las UP cuentan con menos de tres personas encargadas del establo, por lo que no se realiza una gestión administrativa adecuada, evidenciando la falta de registros productivos, reproductivos y económicos.

En el Valle de Tulancingo se reporta que las UP cuentan con una a tres hectáreas (57.1\%), Méndez-Cortés (2019) reporta que la superficie dedicada al pastoreo en promedio fue de 74 hectáreas, en Tabasco las superficies de las UP son de 47 hectáreas en promedio (Granados-Rivera, 2018), por lo que las UP son pequeños establos cerca de las zonas urbanas. En nuestra investigación el $42.9 \%$ de las UP del Valle cuentan con una superficie de pastos entre una y tres hectáreas.

\section{Caracterización de UP bovinas lecheras}

Los productores cuentan con hatos de ganado lechero con alrededor de 20 animales en las UP, lo cual es menor a lo reportado por Granados-Rivera (2018) en donde los promedios en el número de vacas totales son de $39.5 \pm 24.7$ animales. En el Valle de Tulancingo se registró un $39.3 \%$ de vacas en producción con bovinos de raza Holstein y diferentes cruzas con Pardo Suizo o Jersey (46.4\%). Un dato interesante fue que el $85.7 \%$ de la UP mantiene menos de 10 machos en la UP. La ordeña se realiza una vez al día en la mayoría de las UP (85.7\%). La realización del ordeño por lo general inicia a las 6:00 am, con una duración aproximada de tres horas. La producción promedio por vaca es de 6.2 litros de leche al día, al mismo tiempo en que se desteta el becerro para su venta o engorda dentro de la explotación. Datos similares son reportadas por Magaña et al. (2006) para este tipo de sistemas.

El principal tipo de alimentación (50\%) de la UP del Valle de Tulancingo es la mezcla de forrajes, esto puede ser debido a la cercanía de las zonas urbanas, comparado con Tabasco en donde el $98 \%$ de las UP la alimentación del ganado se realiza con base en el pastoreo rotacional en praderas con gramíneas forrajeras de tipo rastrero y amacollado (Granados-Rivera, 2018). El 73.3\% de la ganadería de leche utilizan el pastoreo alterno en gramíneas nativas e introducidas como forma de alimentación 
del hato lechero. El resto (26.7\%) combina el pastoreo tradicional con estrategias alternas a base de residuos de cosechas, desperdicios de la agroindustria y suplementación de sales minerales, práctica que se hace común en la época crítica de forrajes y estiaje. Esto concuerda en términos generales con el manejo alimenticio para los sistemas de bovinos de leche reportado por Ruíz et al. (2008) y Magaña et al. (2006).

Respecto al estado sanitario del ganado del presente estudio mencionan que el $82.1 \%$ de los productores coincide que la presencia de mastitis es una de las principales enfermedades prevalentes en la UP, seguida por la Brucelosis (10.7\%). En este sentido, en Tabasco se reporta que las principales enfermedades que se registran en los bovinos de las UP son diarreas (64\%), mastitis (52\%) y neumonías $(43 \%)$, así mismo, se presentan casos de rabia $(26 \%)$, retención de placenta $(21 \%)$, abortos (12\%), estomatitis (12\%) y gabarro (10\%) (Granados-Rivera, 2018). Se han realizado varios trabajos en otras regiones del país sobre la frecuencia de enfermedades en el ganado bovino lechero (Rosete et al., 2018; Segura et al., 2010; Segura et al.,2003). Se ha encontrado una baja frecuencia, con evidencia serológica, de la presencia de Brucella (Gutiérrez-Hernández et al., 2020). Los resultados del estudio y lo reportado para otros Estados, son similares a los que reporta el Servicio Nacional de Sanidad, Inocuidad y Calidad Agroalimentaria (SENASICA, 2014). En contraste, la prevalencia que reportan algunos autores en hatos lecheros de tipo intensivo, ubicados en zonas endémicas de esta enfermedad, son mucho más altos, influenciadas por el hacinamiento, falta de áreas exclusivas de parto, entre otras que favorecen la transmisión de la bacteria (Milián et al., 2016).

Respecto a la ordeña el $50 \%$ de las UP utiliza una máquina de ordeño, lo cual fue exactamente igual a lo reportado en Tabasco, en donde también el resultado para el sistema de ordeño manual fue de $50 \%$ y mecánico de $50 \%$, la diferencia radica en que los productores se apoyan en el becerro para estimular la eyección de la leche (20\%) (Granados-Rivera, 2018). En las UP del Valle de Tulancingo el número de personas responsables de la ordeña varía entre dos y cinco personas (57.1\%). Dado que la relación entre el trabajador y las vacas lecheras tiene consecuencias directas sobre el bienestar animal y la producción, es importante considerar los rasgos característicos de la personalidad del operario, el grado de satisfacción laboral y de empatía para con los animales; ya que éstos han demostrado ser claves en el tipo de interacción que se construye (Hanna et al., 2009), principalmente cuando el número de personas es bajo dentro del hato.

En cuanto a la asesoría técnica ofrecida a los productores del Valle de Tulancingo fueron similares en su tipo a las detectadas en las UP de Chiapas, pero difieren en su frecuencia (Orantes-Zebadúa et al., 2014). En las UP con bajo nivel de asistencia técnica la frecuencia de enfermedades se incrementa y se desconoce su tipo y frecuencia (Juárez-Barrientos et al., 2015; Vilaboa-Arroniz et al., 2009). En México, más del $70 \%$ de los abortos se considerados de origen desconocido, aunado a estos problemas se suman los de salud, que comprometen la productividad eficiente de los 
animales (Gutiérrez-Hernández et al., 2020; Escamilla et al., 2007). Dentro de las enfermedades más importantes están aquellas que afectan la reproducción, poniendo en riesgo la disponibilidad de becerros; además aumentan el costo de la producción por concepto de tratamientos (Rojo et al., 2009).

En promedio los productores tienen $2.5 \pm 2.5$ años de recibir asistencia técnica y capacitación en el estado de Tabasco (Granados-Rivera, 2018), es importante incorporar este tipo de apoyos a los productores de sistemas de producción de bovinos de leche para el Valle de Tulancingo. El $90 \%$ de los productores expresaron en la encuesta realizada que están a favor de recibir capacitación, principalmente en temas de nutrición, reproducción y prevención de enfermedades.

La amplia variación en las variables que determinan los aspectos socioeconómicos y tecnológicos de las UP explican que algunos productores dediquen parte de su tiempo en actividades productivas no ganaderas, las cuales les permitan obtener ingresos económicos complementarios (Juárez-Barrientos et al., 2015; Oros et al., 2011). En la investigación realizada en Tabasco el $52 \%$ de los productores se dedican exclusivamente a la ganadería y el $48 \%$ restante complementan su ingreso con actividades dentro y fuera de la UP (Granados-Rivera, 2018).

Finalmente, se detectaron problemas que los productores han tenido durante los últimos años por efecto de sequias y en este último año por la pandemia. Tan solo en Veracruz, de enero a septiembre de 2019 se registraron más de 15 mil cabezas de ganado muertas por la sequía (Ruiz et al., 2008). La leche representa un importante ingreso económico en las UP estudiadas debido a que el mayor porcentaje de esta se vende y permite tener un ingreso para cubrir los gastos diarios asociados a las actividades, resultado que coincide con lo reportado en los estados de Chiapas y Veracruz (Juárez-Barrientos et al., 2015; Orantes-Zebadúa et al., 2014). Una situación similar ha sido reportada en la UP localizadas en Chiapas, evidenciando la necesidad de aumentar la capacidad de organización del productor y mejorar el proceso de comercialización del ganado (Orantes-Zebadúa et al., 2014).

\section{CONCLUSIÓN}

Los resultados del presente estudio nos permiten concluir que los productores de ganado bovino lechero del Valle de Tulancingo, presentan un perfil socioeconómico medio, con escolaridad de nivel superior alto. Las prácticas realizadas por los productores de la región son eficientes, aunque requieren de asesoría técnica e información sobre los aspectos nutricionales y reproductivos, así como sobre el manejo sanitario del ganado bovino lechero. 


\section{LITERATURA CITADA}

AGRESTI A. 2013. Categorical data analysis. Stat Papers. 57:49-850. ISSN: 09325026. https://doi.org/10.1007/s00362-015-0733-8

ARIAS RA, Mader TL, Escobar PC. 2008. Factores climáticos que afectan el desempeño productivo del ganado bovino de carne y leche. Archivos de medicina veterinaria. 40(1): 7-22. ISSN 0301-732X. https://dx.doi.org/10.4067/S0301$732 \times 2008000100002$

BERLINGER N, Wynia M, Powell T, Hester M, Milliken A, Fabi R, Cohn F, GuidryGrimes LK, Watson JC, Bruce L, Chuang EJ, Oie G, Abbott J, Jenks PJ. 2020. Ethical framework for Health Care institutions responding to novel Coronavirus SARS-CoV-2 (COVID-19). Guidelines for institutional Ethics services responding to COVID-19. New York: Hastings Center. thehastingscenter.org/ethicalframeworkcovid19

CARRERA CB, Bustamante LTI. 2013. ¿Es la ganadería bovina de carne una actividad competitiva en México? Nóesis. Revista de Ciencias Sociales y $\begin{array}{llll}\text { Humanidades. } & \text { 22(43-1): } 19-50 . & \text { ISSN: }\end{array}$ https://www.redalyc.org/pdf/859/85927874002.pdf

CERVANTES-ESCOTO F, Cesín VA, Mamani OI. 2013. La calidad estándar de la leche en el estado de Hidalgo, México. Revista mexicana de ciencias pecuarias. 4(1): 75-86. ISSN: 2007-1124. https://www.redalyc.org/pdf/2656/265625754008.pdf

CHALATE-MOLINA H, Gallardo-López F, Pérez-Hernández P, Lang-OValle FP, Ortega-Jiménez E, Vilaboa-Arroniz J. 2010. Características del sistema de producción bovinos de doble propósito en el estado de Morelos, México. Zootecnia Tropical. 28 :329-339. ISSN:

0798-7269. http://ve.scielo.org/scielo.php?script=sci_arttext\&pid=S0798-72692010000300004

CORONEL de RM, Ortuño PSF. 2005. Tipificación de los sistemas productivos agropecuarios en el área de riego de Santiago del Estero, Argentina. Revista Latinoamericana de Economía. 36:63-88. ISSN: 0301-7036. http://www.scielo.org.mx/scielo.php?script=sci_arttext\&pid=S030170362005000100004

CUEVAS RV, Baca del Moral J, Cervantes FE, Aguilar JA, 2012. Asistencia técnica en el sector agropecuario en México: análisis del VIII censo agropecuario y forestal. Revista mexicana de ciencias agrícolas. 3:943-957. ISSN: 2007-0934. http://www.scielo.org.mx/scielo.php?script=sci_arttext\&pid=S200709342012000500008 
DÍAZ-RIVERA P, Oros-Noyola V, Vilaboa-Arroniz J, Martínez-Dávila JP, TorresHernández G. 2011. Dinámica del desarrollo de la ganadería doble propósito en las Choapas, Veracruz, México. Tropical and Subtropical Agroecosystems. 14 :191-199. ISSN: 1870-0462. https://www.redalyc.org/pdf/939/93915703018.pdf

ESCAMILLA HP, Martínez MJ, Medina MC, Morales SE. 2007. Frequency and causes of infectious abortion in a dairy herd in Queretaro, Mexico. Canadian Journal of Veterinary Research. 71(4):314-317. http://dx.doi.org/10.21929/abavet2020.22

ESPINOSA V, Rivera G, García L. 2007. Utilidades económicas generadas por la lechería familiar. Sociedades Rurales, Producción y Medio Ambiente. 7:14. ISSN: 1665-1189. https://biblat.unam.mx/es/revista/sociedades-rurales-produccion-ymedio-ambiente/articulo/utilidades-economicas-generadas-por-la-lecheria-familiar

FUENTES LR, Palma AE, Jara-Rojas R. 2012. Factores que influyen en la adopción de tecnologías de conservación de suelos en el secano interior de Chile Central. Revista de la Facultad de Ciencias Agrarias de la Universidad Nacional de Cuyo. 44(2): 31-45. ISSN: 0370-4661. https://www.redalyc.org/pdf/3828/382837651021. pdf

GALINDO-GONZÁLEZ G. 2001. Uso de innovaciones en el grupo de ganaderos para la validación y transferencia de tecnología en "Joachin", Veracruz, México. Tierra Latinoamericana. 19:385-392. ISSN: 2395-8030. http://www.scielo.org.mx/scielo.php?script=sci_arttext\&pid=S187054722016000200237

GRANADOS-RIVERA LD, Quiroz-Valiente J, Maldonado-Jáquez JA, GranadosZurita L, Díaz-Rivera P, Oliva-Hernández J. 2018. Caracterización y tipificación del sistema doble propósito en la ganadería bovina del Distrito de Desarrollo Rural 151, Tabasco, México. Acta Universitaria. 28(6):47-57. ISSN:2007-9621. http://dx.doi.org/10.15174/au.2018.1916.

GUTIERREZ-HERNANDEZ J, Palomares-Reséndiz G, Hernández-Badillo E, LeyvaCorona J, Díaz-Aparicio E, Herrera-López E. 2020. Frecuencia de enfermedades de impacto reproductivo en bovinos de doble propósito ubicados en Oaxaca, México. Abanico veterinario. 10: e114. ISSN 2448-6132. http://www.scielo.org.mx/scielo.php?script=sci_arttext\&pid=S2448$61322020000100132 \& \operatorname{lng}=e s \& n r m=i s 0$

HANNA D, Sneddon IA, Beattie VE. 2009. The relationship between the stockperson's personality and attitudes and the productivity of dairy cows. Animal: an international journal of animal bioscience. 3(5): 737-743. https://doi.org/10.1017/S1751731109003991 
JUÁREZ-BARRIENTOS JM, Herman-Lara E, Soto-Estrada A, Ávalos-de la Cruz DA, Vilaboa-Arroniz J, Díaz-Rivera P. 2015. Tipificación de sistemas de doble propósito para producción de leche en el distrito de desarrollo rural 008, Veracruz, México. Revista Científica. 4:317-323. ISSN: 0798-2259. https://www.redalyc.org/pdf/959/95941173007.pdf

LARA-RODRÍGUEZ DA, Vázquez-Luna D. 2020. El COVID-19 y otros retos de las microempresas bovinas en el estado de Veracruz, México. V Congreso Virtual Internacional Desarrollo Económico, Social y Empresarial en Iberoamérica. https://www.eumed.net/actas/20/desarrollo-empresarial/52-el-covid-19-y-otros-retosde-las-microempresas-bovinas.pdf

MAGAÑA J, Ríos G, Martínez J. 2006. Los sistemas de doble propósito y los desafíos en los climas tropicales de México. XIX Reunión de ALPA y la XXXIII Reunión de la Asociación Mexicana de Producción Animal-AMPA. Tampico, México, 26-28 de octubre 2005. http://www.bioline.org.br/pdf?la06019

MÉNDEZ-CORTÉS V, Mora-Flores JS, García-Salazar JA, Hernández-Mendo O, García-Mata R, García-Sánchez RC. 2019. Tipología de productores de ganado bovino en la zona Norte de Veracruz. Tropical and Subtropical Agroecosystems. 22:304-314. ISSN: 1870-0462.

https://www.google.com/url?sa=t\&rct=j\&q=\&esrc=s\&source=web\&cd=\&cad=rja\&uact $=8 \& v e d=2 a h U K E w j Y q p f H h p 3 w A h X E h K 0 K H e 7 v A f g Q F j A A e g Q I A h A D \& u r l=h t t p s \% 3 A$ \%2F\%2Fdialnet.unirioja.es\%2Fdescarga\%2Farticulo\%2F7260447.pdf\&usg=AOvVaw $1 \mathrm{VdFk}$ si00goThEewWXrGT

MILIÁN SF, Hernández OR, Hernández AL, Alvarado IA, Díaz AE, Mejía EF, Palomares RE, Bárcenas RI, Zendejas MH. 2016. Seroprevalence and risk factors for reproductive diseases in dairy cattle in Mexico. Journal of Veterinary Medicine and Animal Health. 8(8):89-98. https://doi.org/10.5897/JVMAH2016.0483

ORANTES-ZEBADÚA MA, Platas-Rosado D, Córdova-Avalos V, De los Santos-Lara MC, Córdova-Avalos A. 2014. Caracterización de la ganadería de doble propósito en una región de Chiapas, México. Ecosistemas y Recursos Agropecuarios. 1(1):49-57. ISSN: 2007-9028. http://www.scielo.org.mx/scielo.php?script=sci_arttext\&pid=S200790282014000100006

OROS V, Díaz P, Vilaboa J, Martínez JP, Torres G. 2011. Caracterización por grupos tecnológicos de los hatos ganaderos doble propósito en el municipio de Las Choapas, Veracruz, México. Revista Científica Facultad de Ciencias Veterinarias de la Luz. 21(1): 57-63. ISSN: 0798-2259. https://www.redalyc.org/articulo.oa?id=95918054010 
OTZEN T, Manterola C. 2017. Técnicas de Muestreo sobre una Población a Estudio. International Journal of Morphology. 35(1): 227-232. ISSN 0717-9502. https://dx.doi.org/10.4067/S0717-95022017000100037

ROJO RR, Vázquez A JF, Pérez HP, Mendoza MGD, Salem MAZ, Albarrán PB, González RA, Hernández MJ, Rebollar RS, Cardoso JD, Dorantes CEJ, Gutiérrez CJG. 2009. Dual Purpose cattle production in Mexico. Tropical Animal Health and Production. 41:715-721. https://doi.org/10.1007/s11250-008-9249-8

ROSETE FJ, Utrerab RA, Martínez ZJ, Jenkinsa OS, Zuritac GL, Islasa FA, Banda RB, Socci EG. 2018. Prevalencia de anticuerpos contra diarrea viral bovina en vacas no vacunadas en los estados de Puebla, Tabasco y Veracruz, México. Revista Mexicana de Ciencias Pecuarias. 9(3):1-10. ISSN 2448-6698. http://www.scielo.org.mx/scielo.php?script=sci_arttext\&pid=S200711242018000300555\#: :text=La\%20presencia\%20de\%20anticuerpos\%20se,54.6\% $20 \mathrm{y} \% 2076.0 \% 20 \% 25 \% 2 \mathrm{C} \% 20$ respectivamente

RUIZ C, Ávila C, García L, Brunett L. 2008. Sustentabilidad financiera: El caso de una empresa ganadera de bovino de doble propósito. Revista Mexicana de Agronegocios. https://agris.fao.org/agris-search/search.do?recordID=US2016206989

SAGARPA. 2006. Centro de Estadística Agropecuaria -Secretaría de Agricultura, Ganadería y Desarrollo Rural. Sistema de Información Agropecuaria de Consulta. Versión 1.1., México. https://www.gob.mx/agricultura

SALAS-GONZÁLEZ JM, Leos JA, Sagarnaga LM, Zavala-Pineda MJ. 2013. Adopción de tecnologías por productores beneficiarios del programa de estímulos a la productividad ganadera (PROGAN) en México. Revista Mexicana de Ciencias $\begin{array}{llll}\text { Pecuarias. } & \text { 4(2): 243-254. 2007-1124. }\end{array}$ http://www.scielo.org.mx/scielo.php?script=sci_arttext\&pid=S200711242013000200010

SENASICA. Servico Nacional de Sanidad, Inocuidad y Calidad Agroalimentaria. 2014. Dirección general de salud animal. Dirección de campañas zoosanitarias, datos de frecuencias. Servicio Nacional de Sanidad, Inocuidad y Calidad Agroalimentaria. https:/www.gob.mx/senasica/documentos/informes-zoosanitariossemanales-2014

SOLANO C, León H, Pérez E, Herrero M. 2001. Characterizing objective profiles of Costa Rican dairy farmers. Agricultural Systems. 67(3): 153-179. ISSN: 0308-521X. https://doi.org/10.1016/S0308-521X(00)00054-8 
VILABOA J, Diaz P. 2009. Caracterización socioeconómica y tecnológica de los sistemas ganaderos en siete municipios del estado de Veracruz, México. Zootecnia Tropical. 27(4):427-436. ISSN 0798-7269.

http://ve.scielo.org/scielo.php?script=sci_arttext\&pid=S0798-72692009000400008

VILABOA-ARRONIZ J, Díaz-Rivera P, Ruiz-Rosado O, Platas-Rosado DE, González-Muñoz S, Juárez-Lagunes F. 2009. Caracterización socioeconómica y tecnológica de los agro ecosistemas con bovinos de doble propósito de la región del Papaloapan, Veracruz, México. Tropical and Subtropical Agroecosystems. 10:53-62. ISSN: 1870-0462. https://www.redalyc.org/pdf/939/93911243005.pdf 